\title{
ON HARMONIC TENSORS IN AN ALMOST TACHIBANA SPACE
}

\author{
SATOSHI Kото̄
}

(Received April 15,1961)

1. Introduction. In a compact Kählerian space, a skew-symmetric pure tensor field of type $(0, q)$ is harmonic if and only if it is analytic, [5] [6] $]^{1}$. But if the space is non-Kählerian, an (almost) analytic tensor is not necessarily harmonic.

For this problem, S.Tachibana [4] proved the following

THEOREM. In a compact almost Tachibana space, a necessary and sufficient condition that a vector be covariant almost analytic is that $v_{j}$ and $\widetilde{v}_{j}=\varphi_{j}^{l} v_{l}$ are both harmonic.

In this paper, we shall generalize this theorem to a tensor of type $(0, q)$

MAIN THEOREM. In a compact almost Tachibana space, a necessary and sufficient condition that a skew-symmetric pure tensor $T_{(i)}$ of type $(0, q)$ be almost analytic is that $T_{(i)}$ and $\widetilde{T}_{(j)} \equiv{\boldsymbol{\phi}_{j_{s}}}^{s} T_{j q \ldots s . . . s_{1}}$ are both harmonic.

This main theorem follows from the following two lemmas.

LEMMA $\mathrm{A}^{2)}$. In an almost complex space, if skew-symmetric pure tensors $T_{(j)}$ and $\widetilde{T}_{(j)}$ of type $(0, q)$ are both closed, then they are almost analytic.

LEMMA B. In a compact almost Tachibana space, if a skew-symmetric pure tensor $T_{(j)}$ of type $(0, q)$ is almost analytic, then it is harmonic.

In $\$ 2$ we shall give some well known lemmas concerning an almost analytic tensor in an almost complex space and prove Lemma $A$. In $\$ 3$ we shall deal with an almost Tachibana space (which is called a $K$-space by some writers) and give two lemmas obtained by S.Sawaki [3]. In the last section, we shall prove Lemma $B$.

2. Almost analytic tensors. Let $X_{2 n}$ be a $2 n$-dimensional real differentiable manifold of class $C^{\infty}$, with local coordinate $\left\{x^{i}\right\}$, admitting an almost complex structure defined by the tensor field $\boldsymbol{\varphi}_{i}{ }^{h}$ of type (1.1) satisfying

$$
\boldsymbol{\phi}_{i}{ }^{l} \boldsymbol{\phi}_{l}{ }^{h}=-\delta_{i}{ }^{h}, \quad h, i, \ldots=1,2, \ldots \ldots, 2 n .
$$

1) The numbers in brackets refer to References at the end of the paper.

2) This lemma the author owes to Dr. S.Tachibana. Cf., S.Tachibana [5], p. 213. 
A manifold with such an almost complex structure $\varphi_{i}{ }^{h}$ is called an almost complex space.

A tensor field $T_{(i)} \equiv T_{j_{q} \ldots 1_{1}}$ of type $(0, q)$ is called pure in $j_{s}$ and $j_{t}$ if it satisfies

$$
2^{*} O_{j_{i} j_{s}}^{t s} T_{j_{q} \ldots+\ldots s \ldots j_{1}} \equiv T_{j_{q} \ldots j_{1}}+\phi_{j_{t}}^{t} \varphi_{j_{s}}^{s} T_{j_{q} \ldots t \ldots s \ldots j_{1}}=0,
$$

where we denote $T_{j q \ldots t \ldots s \ldots j_{1}}$ instead of $T_{j_{q} \ldots j_{t+1} t_{t-1} \ldots j_{s+1} s_{s-1} \ldots j_{1}}$, etc.. By a pure tensor we shall mean that it is pure in every pair of its indices.

Next, we shall say that a pure tensor field $T_{(j)}$ of type $(0, q)$ is almost analytic if it satisfies ${ }^{3)}$

$$
\boldsymbol{\varphi}_{h}{ }^{l} \partial_{l} T_{(j)}-\partial_{h} \widetilde{T}_{(i)}+\sum_{s=1}^{q}\left(\partial_{j_{s}} \boldsymbol{\varphi}_{h}{ }^{s}\right) T_{j_{q} \ldots s \ldots j_{1}}=0,
$$

which may be written in the tensor form

$$
\boldsymbol{\phi}_{h}{ }^{l} \Gamma_{l} T_{(j)}-\nabla_{h} \widetilde{T}_{(i)}+\sum_{s=1}^{q}\left(\Gamma_{j_{s}} \boldsymbol{\varphi}_{h}{ }^{s}\right) T_{j_{q} \ldots s \ldots j_{1}}=0,
$$

where $\partial_{j}=\partial / \partial x^{j}$ and $\nabla$ denotes the operator of covariant differentiation with respect to the Riemannian connection.

For the almost analytic tensors the following lemmas are well known.

LEMMA 2.1. (S.Tachibana [5]) In an almost complex space, if a skewsymmetric tensor $T_{(i)}$ of type $(0, q)$ is pure, then $\widetilde{T}_{(j)}$ is also a skew-symmetric pure tensor.

LEMMA 2.2. (S.Tachibana [5] $]^{4)}$ In an almost complex space, if a pure tensor field $T_{(j)}$ of type $(0, \mathrm{q})$ is almost analytic, then so is $\widetilde{T}_{(j)}$.

Lastly, we shall prove Lemma A. Since

$$
\partial_{j_{s}}\left({\phi_{h}}^{s} T_{j_{q} \ldots s \ldots j_{1}}\right)=\left(\partial_{j_{s}} \phi_{h}{ }^{s}\right) T_{j_{q} \ldots s \ldots j_{1}}+{\phi_{h}}^{s} \partial_{j_{s}} T_{j_{q} \ldots s \ldots j_{1},},
$$

we have

$$
\sum_{s=1}^{q}\left(\partial_{j_{s}} \boldsymbol{\varphi}_{h}{ }^{s}\right) T_{j_{q} \ldots s \ldots j_{1}}=\sum_{s=1}^{q} \partial_{j_{s}}\left(\boldsymbol{\varphi}_{h}{ }^{s} T_{j_{q} \ldots s \ldots j_{1}}\right)-\sum_{s=1}^{q}{\boldsymbol{\varphi}_{h}}^{s} \partial_{j_{s}} T_{j_{q} \ldots s \ldots j_{1}} .
$$

Therefore if the tensor is skew-symmetric, by virtue of Lemma 2.1, (2.3) may be written as

$$
\boldsymbol{\phi}_{[h}^{l} \partial_{|l|} T_{\left.j_{q} \ldots j_{1}\right]}-\partial_{[h} \widetilde{T}_{\left.j_{q} \ldots j_{1}\right]}=0
$$

3) For $(p, q)$-type tensors, see S. Tachibana [5] or S. Kotō [1].

4) For $(p, q)$-type tensors, see S. Kotō [2]. 
where the square brackets denote the alternating part. If $T_{(i)}$ is an almost analytic tensor, then by Lemma 2.2 so is $\widetilde{T}_{(j)}$. Thus Lemma A was proved.

3. Almost Tachibana spaces. An almost Tachibana space is first of all an almost complex space and secondly has a Riemannian metric $g_{i n}$ satisfying

$$
\boldsymbol{\varphi}_{i}{ }^{m} \boldsymbol{\varphi}_{h}{ }^{l} g_{m l}=g_{i h}
$$

from which

$$
\boldsymbol{\varphi}_{i h}=-\boldsymbol{\varphi}_{h i},
$$

where $\varphi_{i h}=\phi_{i}{ }^{l} g_{l h}$, and finally has the property that the skew-symmetric tensor $\varphi_{i h}$ is a Killing tensor

$$
\nabla_{j} \boldsymbol{\varphi}_{i h}+\nabla_{i} \boldsymbol{\varphi}_{j h}=0 .
$$

Let $R_{k j i}{ }^{h}$ and $R_{j i}=R_{l j i}{ }^{l}$ be Riemannian curvature tensor and Ricci tensor, respectively, then by Ricci identity we have

$$
\nabla_{m} \nabla l \boldsymbol{\varphi}_{j h}-\nabla_{l} \nabla_{m} \boldsymbol{\varphi}_{j h}=\boldsymbol{\varphi}_{h}{ }^{s} \boldsymbol{R}_{m l j s}-\boldsymbol{\varphi}_{j}{ }^{s} \boldsymbol{R}_{m l h s} .
$$

Recently, S. Sawaki [3] proved the following two lemmas

LEMMA 3.1. In an almost Tachibana space, a pure tensor $T_{(j)}$ is almost analytic if and only if

$$
\begin{aligned}
& \nabla_{h} T_{(\jmath)} \text { is a pure tensor, } \\
& \left(\nabla_{j_{s}} \boldsymbol{\varphi}_{h}{ }^{s}\right) T_{j_{q} \ldots s_{\ldots j_{1}}}=0, \quad s=1,2, \ldots \ldots, q .
\end{aligned}
$$

LEMMA 3.2.5) In a compact almost Tachibana space, a necessary and sufficient condition that a pure tensor $T_{(j)}$ of type $(0, q)$ be almost analytic is that it satisfies

$$
\begin{aligned}
& g^{m l} \nabla_{m} \nabla_{l} T_{(i)}-\sum_{s=1}^{q} R_{j_{s}}{ }^{s} T_{j_{q} \ldots s \ldots j_{1}}=0, \\
& \left(\nabla_{j_{s}} \boldsymbol{\varphi}_{h}{ }^{s}\right) T_{j_{q} \ldots s \ldots j_{1}}=0, \quad s=1,2, \ldots \ldots, q .
\end{aligned}
$$

4. Proof of Lemma B. Let $T_{j_{a} \ldots j_{1}}$ be a skew-symmetric almost analytic tensor. Operating $\nabla^{j_{t}}=g^{s^{\prime s t}} \varsigma_{l}(s \neq t)$ to $(2)$ of Lemma 3.2 and taking account of (3.3) we get

$$
\left(\nabla^{j_{t}} \nabla_{j_{s}} \boldsymbol{\varphi}_{h}^{s}\right) T_{j_{q} \ldots s \ldots j_{1}}=\left(\nabla_{h} \boldsymbol{\varphi}_{j_{s}}^{s}\right)\left(\nabla^{j_{t}} T_{j_{q} \ldots s \ldots j_{1}}\right) .
$$

On the other hand, transvecting (1) of Lemma 3.1 with $g^{h j_{t}}$ it follows that

$$
\nabla^{t} T_{j_{q} \ldots t \ldots j_{1}}=0 .
$$

5) For an almost complex space, see S. Kotō [2]. 
Substituting (4.2) in the right hand $\mathrm{me}$ mber of (4.1) and using (3.4) we find

$$
\left(R_{j_{t} s_{s} t s}-\boldsymbol{\varphi}_{j_{t}}^{b} \boldsymbol{\varphi}_{j_{s}}{ }^{a} R_{b a t s}\right) T^{j_{4} \ldots t \ldots s \ldots j_{1}}=0,
$$

where $T^{j_{a} \ldots j_{1}}=T_{j_{q} .{ }_{1} i_{1}} g^{i_{q} j_{a} \cdots} g^{i_{1} j_{1}}$, so that we have

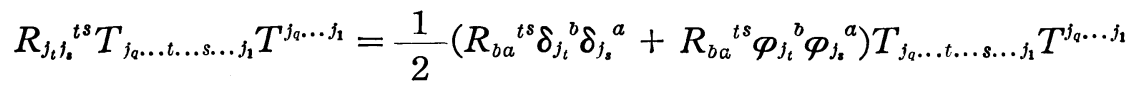

$$
\begin{aligned}
& =R_{j_{t} t_{s}}{ }^{t s} T_{j_{q} \ldots t \ldots s \ldots j_{1}} * O_{b}^{t_{b} j_{i}} T^{j_{q} \ldots b \ldots a \ldots j_{1}} .
\end{aligned}
$$

Since $T_{(j)}$ is a pure tensor, we find

$$
R_{i_{t} j_{s}}^{t s} T_{j_{q} \ldots t \ldots s \ldots j_{1}} T^{j_{q} \ldots j_{1}}=0 .
$$

Consequently, for an almost analytic tensor from (1) of Lemma 3.2 and (4. 3), it follows that

$$
\left(\Delta T_{j_{q} \ldots j_{1}}\right) T^{j_{q} \ldots j_{1}}
$$

$$
\equiv\left(g^{m l} \nabla m \Gamma_{l} T_{(j)}-\sum_{s=1}^{q} R_{s_{s}}^{s} T_{j_{q} \ldots s \ldots j_{1}}-\sum_{t>s} R_{i_{t} j_{s}}{ }^{t s} T_{i_{q} \ldots t \ldots s \ldots j_{1}}\right) T^{j_{q} \ldots j_{1}}=0 .
$$

In the next, it is a well known fact [6] that in a compact orientable Riemannian space $X$, the integral formula

$$
\begin{gathered}
\int_{X}\left[\left(\Delta T_{j_{q} \ldots i_{1}}\right) T^{j_{q} \ldots h_{1}}+(q+1) \nabla^{[h} T^{\left.j_{q} \ldots j_{1}\right]} \nabla[h\right. \\
\left.+q\left(\nabla_{l} T^{j_{q} \ldots j_{2} l}\right)\left(\nabla^{m} T_{i_{q} \ldots j_{2} m}\right]\right) d \sigma=0
\end{gathered}
$$

is valid for any skew-symmetric tensor field $T_{(j)}$ of type $(0, q)$ where $d \sigma$ means the volume element of the $X$.

Hence substituting (4.4) in (4.5), we see that if a skew-symmetric pure tensor $T_{(i)}$ is almost analytic then we have

$$
\nabla\left[h T_{\left.j_{a} \ldots j_{1}\right]}=0 \text {, and } \nabla^{l} T_{j_{s} \ldots j_{2} l}=0,\right.
$$

that is, the tensor becomes harmonic.

q. e. d.

\section{REFERENCES}

[1] S. Kото, Some theorems on almost Kählerian spaces, Jour. of the Math. Soc. Japan, $12(1960), 422-433$.

[2] S. Кото̄, On almost analytic tensors in almost complex spaces, to appear in Tensor.

[3] S.SAWAKI, On almost-analytic tensors of mixed type in a $K$-space, Jour. of the Math. Soc. Japan. 13(1961), 165-182.

[4] S.TACHIBANA, On almost-analytic vectors in certain almost-Hermitian manifolds, Tôhoku Math. Jour., 11(1959), 351-363.

[5] S. TACHIBANA, Analytic tensor and its generalization, Tôhoku Math. Jour., 12(1960), 208-221.

[6] K. YANO and S. BOCHNER, Curvature and Betti numbers. Annals of Math. Studies, $32(1953)$.

NIIGATA UNIVERSITY. 